NASA Technical Memorandum 82766 AIAA-82-0122

\title{
Mode Propagation in Nonuniform Circular Ducts With Potential Flow
}

Y. C. Cho

Lewis Research Center

Cleveland, Ohio

and

K. U. Ingard

Massachusetts Institute of Technology

Cambridge, Massachusetts

JAN 2 1

LANGLEY RESEARCH CENTER

LIBRARY, NASA

HAMPTON, VIRGINIA

Prepared for the

Twentieth Aerospace Sciences Conference sponsored by the American Institute of

- $\quad$ Aeronautics and Astronautics

Orlando, Florida, January 11-14, 1982

\section{NNSA}


MODE PROPAGATION IN NONUNIFORM CIRCULAR DUCTS WITH POTENTIAL FLOW†

Y. C. Cho*

National Aeronautics and Space Administration

Lewis Research Center

Cleveland, Ohio 44135

and

K. U. Ingard**

Massachusetts Institute of Technology

Cambridge, Massachusetts 02139

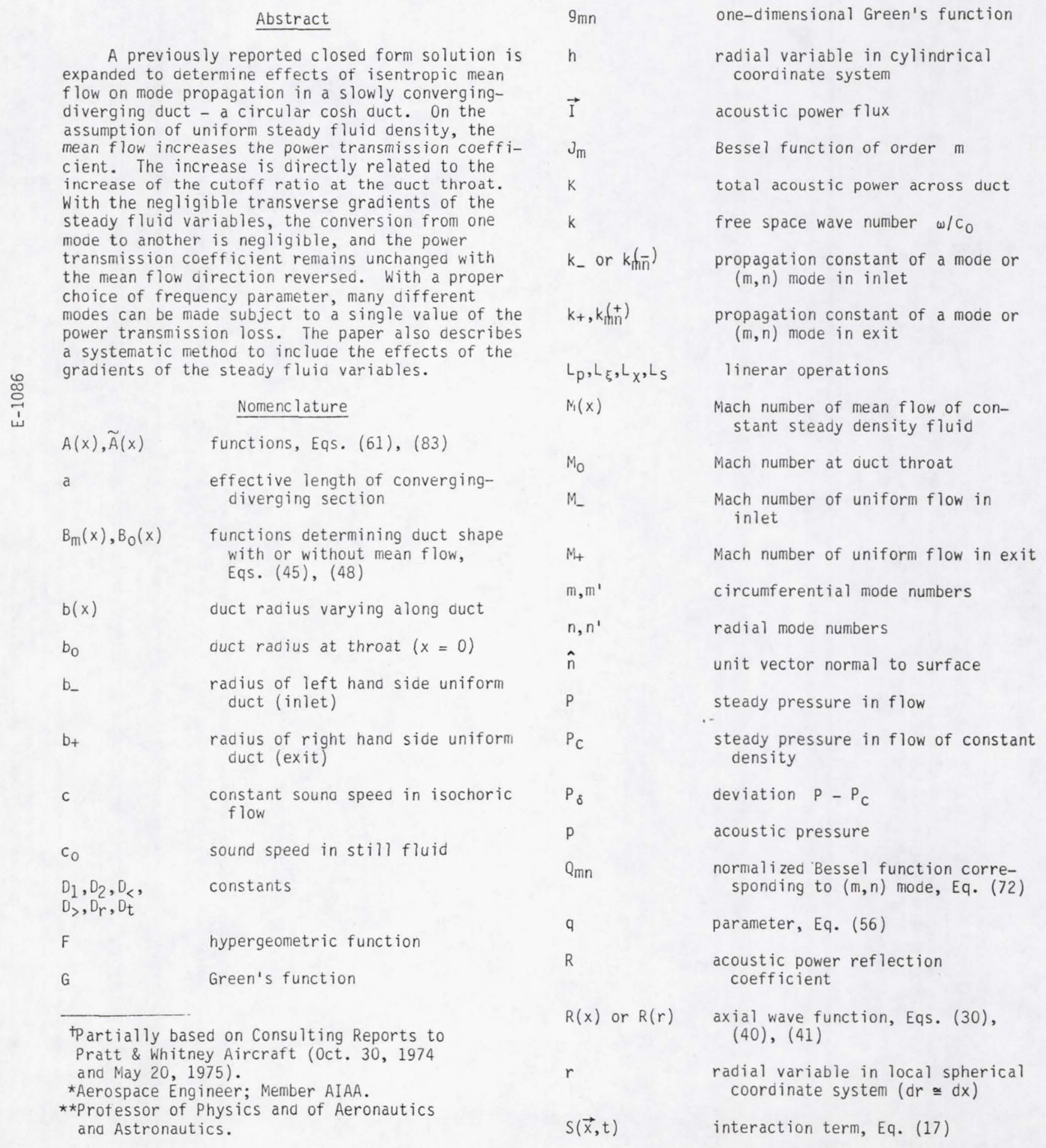




\begin{tabular}{|c|c|}
\hline $\mathrm{S}_{\omega}(\vec{x})$ & $\begin{array}{l}\text { temporal Fourier transform of } \\
S(x, t)\end{array}$ \\
\hline$T$ & $\begin{array}{l}\text { acoustic power transmission } \\
\text { coefficient }\end{array}$ \\
\hline $\mathrm{t}$ & time \\
\hline TL & $\begin{array}{l}\text { acoustic power transmission loss, } \\
\text { Eq. (118) }\end{array}$ \\
\hline$\vec{U}$ & steady velocity of isentropic flow \\
\hline $\overrightarrow{\mathrm{u}}_{\mathrm{c}}$ & $\begin{array}{l}\text { steady velocity of constant density } \\
\text { fluid flow }\end{array}$ \\
\hline$\vec{u}_{\delta}$ & deviation $\vec{U}-\vec{U}_{c}$ \\
\hline$\vec{u}$ & acoustic velocity field \\
\hline$v$ & a constant, Eq. (57) \\
\hline w & a parameter, Eqs. (45) and (46) \\
\hline$w_{0}$ & $w$ with $M=0$ \\
\hline$x^{\prime} s$ & see Eqs. (105) to (109) \\
\hline$x$ & axial coordinate \\
\hline$\vec{x}, \vec{x}_{0}$ & three dimensional coordinates \\
\hline$z$ & $\begin{array}{l}\text { dimensionless axial coordinate, } \\
\text { Eq. (55) }\end{array}$ \\
\hline$\alpha$ or $\alpha_{m n}$ & $\begin{array}{l}\text { eigenvalue corresponaing to a cuct } \\
\text { mode or }(m, n) \text { mode }\end{array}$ \\
\hline$B(x)$ & $b(x) / b_{0}$ \\
\hline$B_{ \pm}$ & $b_{ \pm} / b_{0}$ \\
\hline$\Gamma$ & Gamma function \\
\hline$\gamma$ & specific heat ratio \\
\hline$\gamma_{0}$ & $\begin{array}{l}\text { mode cut-off ratio at auct throat, } \\
\text { Eq. (120) }\end{array}$ \\
\hline$\gamma_{-}$ & $\begin{array}{l}\text { mode cut-off ratio at inlet, } \\
\text { Eq. (119) }\end{array}$ \\
\hline$\delta\left(x-x_{0}\right)$ & Dirac delta function \\
\hline$\delta n, n^{\prime}$ & Kronecher's delta \\
\hline$\varepsilon$ & a parameter, 1 for $M=0$, Eq. (45) \\
\hline$\zeta$ & $\begin{array}{l}\text { a frequency parameter, Eqs. (53) } \\
\text { and (122) }\end{array}$ \\
\hline$n$ & $\begin{array}{l}\text { normalized coordinate variable, } \\
\text { Eq. (32) }\end{array}$ \\
\hline$\theta$ & $\begin{array}{l}\text { polar angle in local spherical } \\
\text { coordinate system }\end{array}$ \\
\hline$\theta_{0}$ & $\begin{array}{l}\text { half cone angle of duct segment, } \\
\text { Fig. } 1\end{array}$ \\
\hline$\mu$ & a parameter, Eq. (47) \\
\hline$\nu$ & frequency parameter, $\mathrm{kb}_{\mathrm{o}} / \alpha$ \\
\hline
\end{tabular}

see Eq. (12)

acoustic perturbation of density spatially varying steady density constant steady density deviation $\rho_{O}-\rho C$

a parameter, Eq. (66)

$b+/ b_{-}$

a Fourier transform of acoustic velocity potential

azimuthal angle variable

see Eq. (13)

acoustic velocity potential

axial wave function, Eq. (41)

angular frequency

\section{Introduction}

In a previously reported investigation of mode propagation in a nonuniform duct, 1 a closed form solution was obtained for a particular class of converging-diverging ducts - circular cosh ducts. These ducts are composed of two asymptotically uniform ducts which are smoothly coupled through a converging-diverging section. The two uniform duct elements can differ from each other in cross sectional area. The duct shape can be adjusted by means of three auct parameters to produce a variety of circular ducts of practical interest. The analysis, which was previously developed for the case of no mean flow, is here expanded to include mean flow effects.

The mean flow under consideration is subsonic, and is assumed to be isentropic. Viscous effects are excluded from discussion. Numerical results will be presented only for cases of low Mach numbers, for which the steady density and pressure hardly vary from one location to another in the duct. However a systematic method is presented to treat finite deviations of the steady fluid variables from the flow of constant density.

\section{Wave Equation}

As customary, the fluid variables are first decomposed into steady and fluctuating parts. The steady part will be further decomposed to treat its spatial variation. The steady density, pressure and flow velocity are denoted respectively by $\rho_{0}, P$ and $U$; and the fluctuations by $\rho$, $p$ and $u$. Retaining only the first order terms of the fluctuations, one obtains from the continuity equation

$\left(\frac{\partial}{\partial t}+\vec{U} \cdot \vec{\nabla}\right) p+\gamma P \vec{\nabla} \cdot \vec{u}+\frac{\gamma P}{\rho_{0}} \vec{\nabla}_{\rho_{0}} \cdot \vec{u}+(\vec{\nabla} \cdot \vec{U})_{p}=0$. 
And from the Euler's equation

$\left(\frac{\partial}{\partial t}+\vec{U} \cdot \vec{\nabla}\right) \vec{u}+(\vec{u} \cdot \vec{\nabla}) \vec{U}+\frac{1}{\rho_{0}} \vec{\nabla} p-\frac{\vec{\nabla} p}{\gamma \rho_{0} P} p=0$.

(2)

Here $\gamma$ is the specific heat ratio of the fluid, and we have used the adiabatic relation between density and pressure

$$
p=\frac{\gamma P}{\rho_{0}} \rho .
$$

Also used are the zeroth order relations

$$
\begin{aligned}
& \vec{\nabla} \cdot\left(\rho_{0} \nabla\right)=0, \\
& \rho_{0}(\vec{U} \cdot \vec{\nabla}) \vec{U}+\vec{\nabla} P=0
\end{aligned}
$$

The steady variables are, in general, functions of spatial coordinates in a nonuniform cuct with mean flow. The functions may be complicated. However, in a subsonic flow, the major portion of the steady variables may assume simple coordinate dependence, and the deviations from the simple dependence can be small. We now decompose the steady variatles further as follows:

$$
\begin{aligned}
& \rho_{0}=\rho_{C}+\rho_{\delta}, \\
& P=P_{C}+\rho_{\delta}, \\
& U=O_{C}+O_{\delta} .
\end{aligned}
$$

Here $\rho_{C}, P_{C}$ and $\vec{U}_{C}$ are a new set of steady variables which are simple functions of spatial coordinates, and $\rho_{\delta}, P_{\delta}$ and $\vec{U}_{\delta}$ are the deviations. In the present analys is, ${ } C_{C}$ and $P_{C}$ are chosen to be constant and set equal to the steady density and pressure in the left side uniform ouct element $(x<0$, or the inlet). The variable $\vec{U}_{C}$ is the velocity of the constant density flow, and therefore, it is subject to the isochoric flow condition

$$
\text { 古 } \cdot U_{c}=0
$$

On inserting Eqs. (6) to (8) into Eqs. (1) ana (2), one obtains

$$
\begin{gathered}
\left(\frac{\partial}{\partial t}+\vec{U}_{C} \cdot \vec{\nabla}\right) p+\gamma_{C} P_{C} \vec{\nabla} \cdot \vec{u}+\xi=0, \\
\left(\frac{\partial}{\partial t}+\vec{U}_{C} \cdot \vec{\nabla}\right) \vec{u}+(\vec{u} \cdot \vec{\nabla}) \vec{U}_{C}+\frac{1}{\rho_{C}} \vec{\nabla} p+\vec{\nabla}_{x}=0,
\end{gathered}
$$

where

$$
\xi=\vec{\nabla} \cdot\left(p \vec{U}_{\delta}\right)+\gamma P_{\delta} \vec{\nabla} \cdot \vec{u}+\vec{u} \cdot \vec{\nabla} P_{\delta},
$$

$$
x=\vec{U}_{\delta} \cdot \vec{u}-\frac{\rho_{\delta}}{\rho_{C}^{2}}\left(1-\frac{\rho_{\delta}}{\rho_{C}}\right) p+o\left(\rho_{\delta}^{3} p\right) .
$$

With the potential flow condition $\vec{\nabla} \times \vec{u}$, one sets

$$
\vec{u}=\vec{\nabla} \Psi(\vec{x}, t),
$$

where $\psi$ is the acoustic velocity potential. With the substitution of this equation, Eqs. (10) and (11) are combined to yield the equation

$$
\nabla^{2} \Psi-\frac{1}{c^{2}}\left(\frac{\partial}{\partial t}+\vec{U}_{c} \cdot \vec{\nabla}\right)^{2} \Psi=S(\vec{x}, t)
$$

where

$$
\begin{aligned}
& c^{2} \equiv \frac{\gamma P_{c}}{\rho_{C}}=c_{0}^{2}-\frac{\gamma-1}{2} U_{c}^{2}(x=-\infty) \\
& S=\frac{1}{c^{2}}\left\{\left(\frac{\partial}{\partial t}+U_{c} \cdot \frac{\vec{\nabla}}{\nabla}\right) x-\frac{\xi}{\rho_{c}}\right\} .
\end{aligned}
$$

Equation (15) is, strictly speaking, a homogeneous equation for the acoustic field. It is, however, convenient to treat it as an inhomogeneous wave equation with the interaction term $S$. With $S=0$, Eq. (15) is the homogeneous wave equation in a mean flow of constant density and pressure. The term $S$ represents the interaction between the acoustic field and the mean flow field due to the spatial variations $\rho_{\delta}, P_{\delta}$ and $\vec{U}_{\delta}$ of the latter. Since no acoustic energy is created or annihilated, the interaction accounts for the elastic scattering of sound by the spatial nonuniformity of the steady fluid variables.

Equation (15) is linear for the acoustic

field. Thus the temporal Fourier transform yields

$$
\nabla^{2} \Phi(\vec{x})+\frac{1}{c^{2}}\left(\omega+i \vec{U}_{c} \cdot \vec{\nabla}\right)^{2} \Phi(\vec{x})=S_{\omega}(\vec{x})
$$

Here

$$
\begin{gathered}
\phi(\vec{x})=\frac{1}{\sqrt{2 \pi}} \int_{-\infty}^{\infty} \Psi(\vec{x}, t) e^{i \omega t} d t . \\
S_{\omega}(\vec{x})=\frac{1}{c^{2}}\left\{\left(-i \omega+c_{c} \cdot \vec{\nabla}\right) x_{\omega}(\vec{x})+\frac{1}{\rho_{C}} \xi_{\omega}(\vec{x})\right\},
\end{gathered}
$$

with

$$
\begin{aligned}
& x_{\omega}(\vec{x})=\frac{1}{\sqrt{2 \pi}} \int_{-\infty}^{\infty} x(\vec{x}, t) e^{i \omega t} d t, \\
& \xi_{\omega}(\vec{x})=\frac{1}{\sqrt{2 \pi}} \int_{-\infty}^{\infty} \xi(\vec{x}, t) e^{i \omega t} d t .
\end{aligned}
$$




\section{Homogeneous Solutions}

We consider solutions to Eq. (18) first with the interaction term neglected. The wave equation is then

$$
\nabla^{2} \Phi_{H}+\frac{1}{c^{2}}\left(\omega+i \vec{U}_{C} \cdot \vec{\nabla}\right)^{2} \Phi_{H}=0
$$

where the subscript $H$ has been used to indicate the homogeneous solutions. The homogeneous solutions serve two purposes: First, with boundary conditions properly satisfied, they describe mode propagation in the duct with mean flow of uniform density, which can be adequately described by the variables $\rho_{C}, P_{C}$ and $\vec{U}_{C}$. It is likely that potential flow solutions will be close to these variables for low Mach numbers. Secondly, the homogeneous solutions can be usea as basis functions to construct the Green's function. With the help of the latter, Eq. (18) can be systematically solved by means of the iterationperturbation method for cases when the deviations of steady fluid variables are finite but small.2

Let us consider Eq. (23) in a circular duct which is specified in terms of the radius $b(x)$, $x$ being the axial coordinate variable. As in Ref. 1, we consider first a small duct segment and the local spherical coordinates $(r, \theta, \phi)$ as illustrated in Fig. 1. The azimuth $\phi$, the angle variable around the auct axis, is not shown in the figure. The duct segment is so short that it may be regarded locally conical. Thus the slope, $b=a b / d x$, of the duct wall remains constant within the segment. The origin of the local spherical coordinate system is located at a point on the duct axis such that the coordinate surface $\theta=\theta_{0}$ (cone) tangentially contacts the duct wall in the segment. The half cone angle $\theta_{0}$ is related to the wall slobe $b^{\prime}$ as

$$
b^{\prime}=\tan \theta_{0} \text {. }
$$

With the spherical coordinates, the isochoric flow velocity is chosen as

$$
\overrightarrow{\mathrm{t}}_{\mathrm{C}}=\hat{\mathrm{r}} \frac{\Omega}{r^{2}}
$$

where $\Omega$ is a constant with the sign being opposite for converging and diverging sections, and $\hat{r}$ is the radial unit vector. Equation (23) can then be written for the spherical coordinates as

$$
\begin{aligned}
& \frac{1}{r^{2}}\left\{\frac{\partial}{\partial r} r^{2} \frac{\partial}{\partial r}+\frac{1}{\sin \theta} \frac{\partial}{\partial \theta} \sin \theta \frac{\partial}{\partial \theta}\right. \\
& \left.+\frac{1}{\sin ^{2} \theta} \frac{\partial^{2}}{\partial \phi^{2}}\right\} \Phi_{H}+\frac{1}{c^{2}}\left(\omega+\frac{i \Omega}{r^{2}} \frac{\partial}{\partial r}\right)^{2} \Phi_{H}=0
\end{aligned}
$$

This equation can be separated into three ordinary differential equations. With the substitution

$$
\Phi_{H}(\vec{x})=R(r) J(\theta) H(\phi)
$$

one obtains, from Eq. (26)

$$
\begin{aligned}
&\left(\frac{d^{2}}{d \phi^{2}}+m^{2}\right) H=0, \\
& \frac{1}{\sin \theta} \frac{d}{d \theta} \sin \theta \frac{d J}{d \theta}+\left(\frac{\alpha^{2}}{\sin ^{2} \theta_{0}}-\frac{m^{2}}{\sin ^{2} \theta}\right) J=0, \\
&\left(1-M^{2}\right) \frac{d^{2} R}{d r^{2}}+\left[\frac{2\left(1+M^{2}\right)}{r}-2 i k M\right] \frac{d R}{d r} \\
&+\left[k^{2}-\left(\frac{\alpha}{r \sin \theta_{0}}\right)^{2}\right] R=0 .
\end{aligned}
$$

where $m$ and $\alpha$ are the separation constants, and

$$
k=\frac{\omega}{c}, M=\frac{\Omega}{c r^{2}} .
$$

The solution to Eq. (28) is

$$
H=e^{i m \phi}
$$

with $m$ being an integer. As for Eq. (29), it is convenient to introduce the normalized coordinate variable $n_{\text {, }}$, defined as

$$
\begin{aligned}
n & =\frac{\sin \theta}{\sin \theta_{0}} \text { for } \theta_{0} \neq 0 \\
& =\frac{h}{b} \text { for } \theta_{0}=0 .
\end{aligned}
$$

where $h$ is the radial variable in the cylindrical coordinate system. The value of $n$ varies from 0 to 1 in the duct. On the duct axis, $n=0$; and on the duct wall, $n=1$. Equation (30) is written in terms of $n$ as

$$
\begin{array}{r}
\left(1-\sin ^{2} \theta_{0} n^{2}\right) \frac{d^{2} J}{d n^{2}}+\frac{1}{n}\left(1-2 \sin ^{2} \theta_{0} n^{2}\right) \frac{d J}{d n} \\
\cdot . \\
+\left(\alpha^{2}-\frac{m^{2}}{n^{2}}\right) J=0 .
\end{array}
$$

For a duct of slowly varying cross section $\left(\left|b^{\prime}\right| \ll 1\right)$, sin $\theta_{0} \cong b^{\prime}$. Neglecting terms involving $\left(b^{\prime}\right)^{2}$ and higher orders, one can write Eq. (33) as

$$
\frac{d^{2} J}{d n^{2}}+\frac{1}{n} \frac{d J}{d n}+\left(\alpha^{2}-\frac{m^{2}}{n^{2}}\right) J=0 .
$$

This is the Bessel equation of order $m$, and the physically acceptable solution is

$$
J \sim J_{m}(\alpha n)
$$

where $J_{m}$ is the Bessel function of order $m$. From the boundary condition of the hard duct wall, one determines values of a as follows: 


$$
\alpha=\alpha_{m n}, n=0,1,2 \ldots
$$

where $\alpha_{m n}$ is the $n$-th zero of $d J_{m}(x) / d x$.

Some remarks should be made on the coordinate variable $n$. Unlike the variable $\theta, n$ is not a local coordinate variable, but it is valid through the entire duct. To see this, let us consider two small duct segments 1 and 2, respectively at $x=x_{1}$ and $x_{2}$. The half cone angles of the respective segments are denoted by ${ }^{\theta} 0,1$ and ${ }^{\theta} 0,2$. Assume ${ }^{\theta} 0,1>{ }^{\theta} 0,2$. The variable $\theta$ varies from' 0 to $\theta_{0}, 1$ in segment 1 , and from 0 to ${ }^{\theta} 0,2$ in segment 2 . A value of $\theta$ greater than $\theta 0,2$ and less than ${ }^{\theta} 0,1$, represents a coordinate surface in segment 1 , but not in segment 2 . Furthermore, two coordinate surfaces represented by a value of $\theta$ less than $\theta_{0,2}$, respectively in the two duct segments are not, in general, equivalent to each other. On the other hand, $n$ varies from 0 to 1 for all the duct segments. The locations represented by a value of $n$ are in one-to-one correspondence for two different duct segments. A coordinate surface is uniquely determined as the locus of $n=\eta_{1}, n_{1}$ being a constant, and the coordinate surface spans all the duct segments. A coordinate surface with a value of $n$ does not intersect with any other coordinate surface with a different value of $n$. The duct wall is a coordinate surface corresponding to $\eta=1$. We also note, from Eqs. (35) and (36), that an eigenfunction assumes the same value on a coordinate surface $n=n 1$. A mode is in oneto-one correspondence with an eigenfunction. Thus, with the use of the variable $n$, a mode is defined in a nonuniform circular duct with isochoric flow if $\left|b^{\prime}\right|$ remains small (Fig. 2).

Equation (30) governs the propagation of a mode along the duct. The equation is first transformed from the local coordinate $r$ to the duct coordinate $x$. To this end we use the relations

$$
\begin{aligned}
& \sin \theta_{0}=b^{\prime} \\
& r=\frac{b}{b^{\prime}} \\
& \frac{d}{d r}=\frac{d}{d x}
\end{aligned}
$$

These relations are valid for all the duct segments and the error is of order $\left(b^{\prime}\right)^{2}$. In order to avoid misunderstandings, a remark should be made on Eq. (39): Eq. (30) governs the wave along a line that is the intersection of coordinate surfaces $\phi=$ constant and $n=$ constant (not

$\theta=$ constant). On this line, $d r=d x \sqrt{1+b^{12}}$, and Eq. (39) follows. One should not attempt to derive a similar relation by regarding $r$ as a function of $h$ as well as $x$. Such a derivation micht lead one to an incorrect conclusion, and the error would appear to be of order $b^{\prime}$ instead of $\left(b^{\prime}\right)^{2}$. Note that the oriain of the local coordinate system changes with $x$. It cannot be overemphasized that $R$ (as given in Eq. (30)) deals with the wave field variation along a line which is the intersection of coordinate surfaces $\phi=$ constant and $n=$ constant. Finally, the use of the relations (37), (38), and (39) is very unlikely to lead one to any asymptotic paradox.
Using Eqs. (37) to (39), one can write Eq. (30) as

$$
\begin{aligned}
\left(1-M^{2}\right) \frac{d^{2} R}{d x^{2}}+\left[\frac{2 b^{\prime}}{b}(1\right. & \left.\left.+M^{2}\right)+2 i k M\right] \frac{d R}{d x} \\
& +\left(k^{2}-\frac{a^{2}}{b^{2}}\right) R=0 .
\end{aligned}
$$

Here we have used

$$
\begin{aligned}
& M(x)=\frac{M_{0}}{\beta^{2}(x)} \\
& B(x)=\frac{b(x)}{b_{0}}
\end{aligned}
$$

where $b_{0}$ and $M_{0}$ are the duct radius and the Mach number at the duct throat. For a given $B(x), M(x)$ is determined once $M_{0}$ is known. Thus, for a given duct shape, $M_{0}$ is sufficient to specify $M(x)$.

With the substitution

$$
R(x)=\frac{\psi(x)}{b(x) \sqrt{1-M^{2}(x)}} \exp \left(-i k \int^{x} \frac{M(x)}{1-M^{2}(x)} d x\right) \text {, }
$$

Equation (40) is transformed into

$$
\frac{d^{2} \psi}{d x^{2}}+\left[\left(\frac{k}{1-M^{2}}\right)^{2}-\frac{1}{1-M^{2}} \frac{\alpha^{2}}{b^{2}}\right] \psi=0,
$$

where terms like $b^{\prime 2}$ and $b^{\prime \prime}$ have been neglected.

\section{Solutions in a Circular Cosh Duct}

Equation (42) was solved exactly in a closed form with a circular cosh duct when $M=0$, in Ref. 1. With $M \neq 0$, it is convenient to introduce a function $B_{M}(x)$ as follows:

$$
B_{M}(x)=\frac{1}{\left(1-M^{2}\right) B^{2}}-v^{2} \frac{M^{2}\left(2-M^{2}\right)}{\left(1-M^{2}\right)^{2}}
$$

where $v=k b_{0} / \alpha$. Note that this function depends on the Mach number and the frequency $(v)$ as well as the duct shape $(B(x))$. With the use of Eq. (43), (42) is written as

$$
\frac{d^{2} \psi}{d x^{2}}+\left[k^{2}-\frac{a^{2}}{b_{0}^{2}} B_{M}(x)\right] \psi=0
$$

The discussions thus far apply to any nonuniform hardwal1 circular duct as long as $b^{\prime 2}$ and b" are negligible.

To utilize the cosh duct solution, we set 


$$
\begin{aligned}
& B_{M}(x)=B_{M}(0)+w\left\{\cosh ^{2} \mu \operatorname{sech}^{2}(\varepsilon x / a-\mu)\right. \\
& -\sinh (2 \mu) \tanh (\varepsilon x / a-\mu)-\cosh (2 \mu)\} .
\end{aligned}
$$

Here $a$ is the effective length of the converging-diverging section, and $w, \mu$ and $\varepsilon$ are parameters depending on $M, v$ ana duct geometry. The parameters $W$ and $\mu$ are determined as

$$
\begin{gathered}
w=\left\{\left[B_{M}(0)-B_{M}(\infty)\right]\left[B_{M}(0)-B_{M}(-\infty)\right]\right\}^{1 / 2}, \\
\mu=\frac{1}{4} \ln \left\{\frac{B_{M}(0)-B_{M}(+\infty)}{B_{M}(0)-B_{M}(-\infty)}\right\} .
\end{gathered}
$$

Involved in these equations are $M, \nu$ and $B_{ \pm}$ the radius ratios of the duct. For fixea values of $M_{0}$ and $v, W$ and $\mu$ are determined from the radius ratios $B_{ \pm}$, and vice versa. One notices from Eq. (47) that $\mu$ is equal to zero for a symmetric duct $\left(\beta_{+}=\beta_{-}\right)$, regardless of values of $M_{0}$ and $v$.

The parameter $\varepsilon$ is not given in a closed form, but its value can be determined analytically. For fixed values of $M_{0}$ and $v$, the duct radius ratios $\beta_{ \pm}$have been used for the determination of $w$ and $\mu$. The axial variation of the radius in the converging-diverging section will be used to determine $\varepsilon$. If the value of $\varepsilon$ were kept constant, the duct shape would depend on $M$ and $v$ as can be readily seen from Eqs. (43) and (45). Thus, the value of $\varepsilon$ must be so oetermined that the duct shape may remain unchanged for different values of $M$ and $v$. To this end, at a properly chosen axial location in the converging-diverging section, the duct radius is required to be the same for all the allowed values of $M_{0}$ and $v$. We choose $M_{0}=0$ as the reference case. With $M_{0}=0$, we set $\varepsilon=1$, and obtain from Eq. (43)

$$
\left.B_{0}(x) \equiv B_{M}(x)\right|_{M=0}=\beta^{-2}(x),
$$

and from Eq. (45)

$$
\begin{aligned}
& B_{0}(x)=1+w_{0}\left\{\cosh ^{2} \mu_{0} \operatorname{sech}^{2}\left(x / a-\mu_{0}\right)\right. \\
& \left.-\sinh \left(2 \mu_{0}\right) \tanh \left(x / a-\mu_{0}\right)-\cosh \left(2 \mu_{0}\right)\right\}
\end{aligned}
$$

where the subscript 0 indicates $M=0$. Note that $B_{0}$, wo and $\mu_{0}$ do not depend on $v$. The axial location is chosen preferably in the converging section, as $x=-s$. The duct radius at that location is determined from Eqs. (48) and (49):

$B(-s)=\left\{1+w_{0} \cosh ^{2} \mu_{0} \operatorname{sech}^{2}\left(s / a+\mu_{0}\right)\right.$

$\left.\sinh \left(2 \mu_{0}\right) \tanh \left(s / a-\mu_{0}\right)-\cosh \left(2 \mu_{0}\right)\right\}^{1 / 2}$

With $M_{0} \neq 0$, Eq. (43) is written for $x=-s$ as
$B_{M}(-S)=\frac{1}{\left[1-M^{2}(-s)\right] B^{2}(-s)}-v^{2} \frac{M^{2}(-s)\left[2-M^{2}(-s)\right]}{\left[1-M^{2}(-s)\right]^{2}}$

and Eq. (45) is

$$
\begin{aligned}
& B_{M}(-s)=B_{M}(0)+w\left\{\cosh ^{2} \mu \operatorname{sech}^{2}(\varepsilon s / a+\mu)\right. \\
& +\sinh (2 \mu) \tanh (\varepsilon s / a+\mu)-\cosh (2 \mu)\}
\end{aligned}
$$

The value of $\varepsilon$ is determined from Eqs. (50) to (52). There may exist more than one real solution for $\varepsilon$; the correct one should be determined from physical considerations. A list of values of $\varepsilon$ are given in Table 1, for various values of $M_{0}$ and $\zeta$, where $\zeta$ is a frequency parameter defined as

$$
\zeta=\frac{1}{1-M_{0}^{2}}\left(k b_{0}-\alpha \sqrt{1-M_{0}^{2}}\right)
$$

As the table shows, $\varepsilon$ is close to unity in most cases of interest. Also notice that $\varepsilon$ takes the same value for all the modes at $\zeta=0$. The value of $\varepsilon$ at $\zeta=0$ are listed for various values of $M_{0}$ and $B_{-}$in Table 2 .

We now return to Eq. (44). On inserting Eq. (45) into Eq. (44) we obtain

$$
\begin{aligned}
& \frac{d^{2} \psi}{d z^{2}}+\left[q^{2}-v\left(\cosh ^{2} \mu \operatorname{sech}^{2} z\right.\right. \\
& \quad-\sinh (2 \mu) \tanh z)] \psi=0
\end{aligned}
$$

where

$$
z=\varepsilon \times / a-\mu
$$

$$
q^{2}=(k a / \varepsilon)^{2}-\left(\alpha a / \varepsilon b_{0}\right)^{2}\left[B_{M}(0)-w \cosh (2 \mu)\right] .
$$

$$
v=\left(\alpha a / \varepsilon b_{0}\right)^{2} w
$$

Equation (54) can be solved exactly.2 The general solution is written as

$$
\psi=D_{1} \psi_{1}(z)+D_{2} \psi_{2}(z)
$$

Here $D_{1}$ and $D_{2}$ are constants; and $\psi_{1}$ and $\psi_{2}$ are linearly independent solutions.

$$
\begin{aligned}
& \psi_{1}=A(z) F\left(\zeta_{1}, \zeta_{2}, \zeta_{3} ;\left(1+e^{2 z}\right)^{-1}\right), \\
& \psi_{2}=A(z)\left(1+e^{2 z}\right)^{-i k_{+} a / \varepsilon} .
\end{aligned}
$$


$F\left(\zeta_{2}-\zeta_{3}+1, \zeta_{1}-\zeta_{3}+1,2-\zeta_{3} ;\left(1+e^{2 z}\right)^{-1}\right)$,

where $F$ is the hypergeometric function, and

$$
\begin{aligned}
& A(z)=e^{i\left(k_{+} k_{-}\right) z a / 2 \varepsilon}(2 \cosh z)^{i\left(k_{+}+k_{-}\right) a / 2 \varepsilon}, \\
& k_{ \pm}=\frac{k}{1-M_{ \pm}^{2}}\left[1-\left(1-M_{ \pm}^{2}\right)\left(\frac{\alpha}{k b_{ \pm}}\right)^{2}\right]^{1 / 2}, \\
& b_{ \pm}=b(x= \pm \infty), M_{ \pm}=M(x= \pm \infty), \\
& \zeta_{1}=\left\{1-i\left[\left(k_{+}+k_{-}\right) a / \varepsilon+\sigma\right]\right\} / 2 \\
& \zeta_{2}=\left\{1-i\left[\left(k_{+}+k_{-}\right) a / \varepsilon-\sigma\right]\right\} / 2 \\
& \zeta_{3}=1-i k_{+} a / \varepsilon \\
& \sigma=\left(4 v \cosh _{\mu}^{2} 1\right)
\end{aligned}
$$

The constants $D_{1}$ and $D_{2}$ are to be determined from bounoary conditions at $x= \pm \infty$. The asymptotic forms of the hypergeometric function are used to find

i) at $x=+\infty$

$$
\begin{aligned}
& \psi_{1}=e^{-i k_{+} \mu a / \varepsilon} e^{i k_{+} x}, \\
& \psi_{2}=e^{i k_{+} \mu a / \varepsilon} e^{-i k_{+} x},
\end{aligned}
$$

ii) at $x=-\infty$

$\psi_{1}=\frac{\Gamma\left(\zeta_{3}\right) \Gamma\left(\zeta_{1}+\zeta_{2}-\zeta_{3}\right)}{\Gamma\left(\zeta_{1}\right) \Gamma\left(\zeta_{2}\right)} e^{-i k_{-} \mu a / \varepsilon+i k_{-} x}$

$$
+\frac{\Gamma\left(\zeta_{3}\right) \Gamma\left(\zeta_{3}-\zeta_{1}-\zeta_{2}\right)}{\Gamma\left(\zeta_{3}-\zeta_{1}\right) \Gamma\left(\zeta_{3}-\zeta_{2}\right)} e^{i k_{-} \mu a / \varepsilon-i k_{-} x},
$$

$$
\begin{aligned}
\psi_{2} & =\frac{\Gamma\left(2-\zeta_{3}\right) \Gamma\left(\zeta_{1}+\zeta_{2}-\zeta_{3}\right)}{\Gamma\left(\zeta_{2}-\zeta_{3}+1\right) \Gamma\left(\zeta_{1}-\zeta_{3}+1\right)} e^{-i k_{-} \mu a / \varepsilon^{+i k_{-}} x} \\
& +\frac{\Gamma\left(2-\zeta_{3}\right) \Gamma\left(\zeta_{3}-\zeta_{1}-\zeta_{2}\right)}{\Gamma\left(1-\zeta_{1}\right) \Gamma\left(1-\zeta_{2}\right)} e^{i k_{-} \mu a / \varepsilon-i k_{-} x} \cdot
\end{aligned}
$$

where $I$ is the Gamma function.
Mode Propagation in Circular Cosh Ducts With Low Mach Number Flow

As mentioned earlier, the deviations $\rho_{\delta}$, $P_{\delta}$ and $\vec{U}_{\delta}$ are negligible in cases of low Mach numbers $\left(M_{0}<0.5\right)$. In these cases, the solutions can be obtained as in Eq. (58), with the boundary conditions to be satisfied. Note that these solutions are the zeroth order solutions to Eq. (18) and can be used to obtain more accurate solutions.

Let us begin with the incident wave that is composed of the $(m, n)$ mode coming from $x=-\infty$ : In the incident side uniform duct $(x<0)$,

$$
\begin{aligned}
\Phi_{I}=Q_{m n}(n) e^{i m \phi} \frac{1}{b_{-} \sqrt{1-M_{-}^{2}}} \\
\quad \times \exp \left[i\left(-k M_{-} /\left(1-M_{-}^{2}\right)+k_{m n}^{(-)}\right) \times\right],
\end{aligned}
$$

where

$$
Q_{m n}(n)=J_{m}\left(\alpha_{m n} n\right) / L_{m n}
$$

$$
L_{m n}=\left\{\int_{0}^{1} J_{m}^{2}\left(\alpha_{m n}\right) n d n\right\}^{1 / 2}
$$

$$
k_{m n}^{( \pm)}=\left.k_{ \pm}\right|_{\alpha=\alpha_{m n}}
$$

Corresponaingly, we have

$$
\psi_{I}=e^{i k_{m n}^{(-)} x} .
$$

With mode conversion neglected, the solution $\psi$ is given at $x \neq \pm \infty$ in the form

$$
\begin{aligned}
& \lim _{x \rightarrow-\infty} \psi(x)=e^{i k_{m n}^{(-)} x}+D_{r} e^{-i k_{m n}^{(-)} x}, \\
& \lim _{x \rightarrow \infty} \psi(x)=D_{t} e^{i k_{m n}^{(+)} x}
\end{aligned}
$$

The second term of Eq. (76) represents the reflected wave, and Eq. (77) the transmitted wave. The constants in Eq. (58) are readily aetermined by comparing the asymptotic expressions in Eqs. (67) to (70) with Eqs. (76) and (77):

$$
D_{2}=0
$$

$$
D_{1}=e^{i k_{m n}^{(-)} \mu a / \varepsilon} \frac{\Gamma\left(\zeta_{1}\right) \Gamma\left(\zeta_{2}\right)}{\Gamma\left(\zeta_{3}\right) \Gamma\left(\zeta_{1}+\zeta_{2}-\zeta_{3}\right)}
$$


The coefficients $D_{r}$ and $D_{t}$ are

$D_{r}=e^{2 i k_{\min }^{(-)} \mu a / \varepsilon} \frac{\Gamma\left(\zeta_{1}\right) \Gamma\left(\zeta_{2}\right) \Gamma\left(\zeta_{3}-\zeta_{1}-\zeta_{2}\right)}{\Gamma\left(\zeta_{1}+\zeta_{2}-\zeta_{3}\right) \Gamma\left(\zeta_{3}-\zeta_{1}\right) \Gamma\left(\zeta_{3}-\zeta_{2}\right)}$,

$D_{t}=e^{i\left(k_{m n}^{(-)}-k_{m n}^{(+)}\right) \mu a / \varepsilon} \frac{\Gamma\left(\zeta_{1}\right) \Gamma\left(\zeta_{2}\right)}{\Gamma\left(\zeta_{3}\right) \Gamma\left(\zeta_{1}+\zeta_{2}-\zeta_{3}\right)}$

In Eqs. (78) to (81) the parameters $\mu, \varepsilon, \zeta 1$, $\zeta_{2}$ and $\zeta 3$ contain the constant $\alpha$ : it is to be replaced by the eigenvalue $\alpha_{m n}$. The solution is

$$
\begin{gathered}
\Phi=Q_{m n}(n) e^{i m \phi} b^{-1}\left(1-M^{2}\right)^{-1 / 2} \\
\quad x \exp \left(i k \int_{0}^{x} \frac{M}{1-M^{2}} d x\right) . \\
\tilde{A}(z) r\left(\zeta_{1}, \zeta_{2}, \zeta_{3} ;\left(1+e^{2 z}\right)^{-1}\right)
\end{gathered}
$$

where

$$
\tilde{A}(z)=D_{1} A(z) \exp \left(i \frac{k M_{-}}{1-M^{2}} x_{-}+i k \int_{x_{-}}^{0} \frac{M}{1-M^{2}} d x\right)
$$

$x_{-}$being a large negative value of $x$. Recall that $n=h / b(x)$ and $z=\varepsilon x / a-\mu$.

\section{Green's Function and General Solutions}

When the interaction term $S_{\omega}$ is finite but small, Eq. (18) can be solved by means of the iteration-perturbation method. 3 To this end, the equation is first replaced by the integral equation

$$
\phi(\vec{x})=\phi^{(0)}(\vec{x})+\int G\left(\vec{x}, \vec{x}_{0}\right) S_{\omega}\left(\vec{x}_{0}\right) d^{3} \vec{x}_{0}
$$

Here $G$ is the Green's function to be discussed, and the function $\phi(0)$ has been used for the homogeneous solution, to let the solution satisfy the boundary conditions.

The equation for the Green's function is

$$
\left\{\nabla^{2}+\frac{1}{c^{2}}(\omega+i \vec{U} c \cdot \vec{\nabla})^{2}\right\} G\left(\vec{x}, \vec{x}_{0}\right)=\delta\left(\vec{x}-\vec{x}_{0}\right)
$$

The solution can be obtained in terms of the homogeneous solutions with proper boundary conditions as follows:

$$
\begin{aligned}
G\left(\vec{x}, \vec{x}_{0}\right)=\frac{1}{2 \pi} & \sum_{m} e^{i m\left(\phi-\phi_{0}\right)} \\
& \times \sum_{n} Q_{m n}(n) Q_{m n}\left(n_{0}\right) g_{m n}\left(x, x_{0}\right)
\end{aligned}
$$

Here $\mathrm{gmn}_{\mathrm{mn}}$ is the one-dimensional Green's function. On inserting Eq. (86) into Eq. (85) and using the orthogonality and the completeness of the eigenfunctions, one obtains

$$
\begin{aligned}
\left\{\left(1-M^{2}\right) \frac{d^{2}}{d x^{2}}+\left[\frac{2 b^{1}}{b}\left(1+M^{2}\right)-2 i k M\right] \frac{d}{d x}\right. \\
\left.+\left(k^{2}-\frac{a_{m n}^{2}}{b^{2}}\right)\right\} g_{m n}\left(x, x_{0}\right)=\delta\left(x-x_{0}\right) .
\end{aligned}
$$

The homogeneous solution of this equation is none other than $R$ given in Eq. (41).

Consider the factor $\psi$ first. For a circular cosh duct, the homogeneous solutions $\psi_{1}$ and $\psi_{2}$ are given in Eqs. (59) and (60). The source term is non-zero only in the converging-diverging section. Thus, the Green's function is required to represent outgoing waves in the uniform duct elements. Consider a new set of homogeneous solutions $\psi_{>}$and $\psi_{<}$which asymptotically behave as

$$
\begin{aligned}
& \psi_{>}(x) \stackrel{x \rightarrow \infty}{\rightarrow} e^{i k_{m n}^{(+)} x}, \\
& \psi_{<}(x) \stackrel{x \rightarrow-\infty}{\longrightarrow} e^{-i k_{m n}^{(-)}} .
\end{aligned}
$$

Comparing these equations with Eqs. (67) to (70), one can readily find

$$
\psi_{>}=\psi_{1},
$$

$\psi_{<}=\psi_{1}-\frac{\Gamma\left(\zeta_{3}\right) \Gamma\left(\zeta_{2}-\zeta_{3}+1\right) \Gamma\left(\zeta_{1}-\zeta_{3}+1\right)}{\Gamma\left(\zeta_{1}\right) \Gamma\left(\zeta_{2}\right) \Gamma\left(2-\zeta_{3}\right)} \psi_{2}$.

Corresponding to these functions, the homogeneous solutions to $\mathrm{Eq}$. (87) are

$$
R_{>}(x)=b^{-1}\left(1-M^{2}\right)^{-1} \exp \left(-i x \int^{x} \frac{M}{1-M^{2}} d x\right) \psi_{>}(x),
$$

$R_{<}(x)=b^{-1}\left(1-M^{2}\right)^{-1} \exp \left(-i k \int^{x} \frac{M}{1-M^{2}} d x\right) \psi_{<}(x)$. 
The one-dimensional Green's function is then obtained as

$g_{m n}\left(x, x_{0}\right)=\frac{1}{x_{0} \Delta\left(x_{0}\right)}\left\{\begin{array}{l}R_{>}(x) R_{<}\left(x_{0}\right) \text { for } x>x_{0} \\ R_{<}(x) R_{>}\left(x_{0}\right) \text { for } x<x_{0}\end{array}\right.$

where $\Delta$ is the Wronskian

$$
\Delta=\left|\begin{array}{ll}
R_{<} & R_{<}^{\prime} \\
R_{>} & R_{>}^{\prime}
\end{array}\right|
$$

The source $S_{\omega}$, as given in Eq. (20) is a linear function of acoustic field variables. The latter are not known until the problem is solved. However, when the deviations, $\rho$, $P_{\delta}$ and $\vec{u}_{\delta}$ are small, the source is small. Thus, the zeroth order solution $\phi(0)$ can be used for the acoustic field variables to compute $S_{\omega}$. The acoustic velocity is $\vec{u}(0)=\vec{\nabla} \phi(0)$, and the acoustic pressure is obtained from Eqs. (10) and (11) with $\xi$ and $x$ neglected.

$$
p^{(0)}=L_{p}{ }^{(0)} \text {, }
$$

where $L_{p}$ is a linear operator defined as

$$
\begin{aligned}
L_{p} & =i \rho_{c} c\left\{\left(\frac{\alpha}{k b}\right)^{2}\right. \\
& \left.-\left[i M_{1}+\frac{2\left(1+M^{2}\right) b^{\prime}}{k b}\right] \frac{\partial}{\partial x}-\frac{1-M^{2}}{k} \frac{\partial^{2}}{\partial x^{2}}\right\} .
\end{aligned}
$$

It follows that

$$
\begin{aligned}
& \xi_{\omega}^{(0)}=L_{\xi}{ }^{\phi}(0), \\
& x_{\omega}^{(0)}=L_{x}{ }^{(0)}
\end{aligned}
$$

Here $L_{\xi}$ and $L_{\chi}$ are linear operators containing the deviations of the mean fluid variables:

$$
\begin{aligned}
& L_{\xi}=\left(\vec{\nabla} \cdot \dot{0}_{\delta}+\dot{0}_{\delta} \cdot \vec{\nabla}\right) L_{p} \\
&-\gamma P_{\delta}\left(\frac{\alpha^{2}}{b^{2}}-\frac{2 b^{\prime}}{b} \frac{\partial}{\partial x}-\frac{\partial^{2}}{\partial x^{2}}\right)+\vec{\nabla}_{\delta} \cdot \vec{\nabla}, \\
& L_{x}=\dot{U}_{\delta} \cdot \vec{\nabla}-\frac{\rho_{\delta}}{\rho_{C}^{2}}\left(1-\frac{\rho_{\delta}}{\rho_{C}}\right) L_{p}-\frac{1-M^{2}}{k} \frac{\partial^{2}}{\partial x^{2}}
\end{aligned}
$$

The source term is then

$$
S_{\omega}^{(0)}=L_{S^{\phi}}(0)
$$

with the operator defined as

$$
L_{S}=\frac{1}{c^{2}}\left\{\left(-i \omega+U_{c} \frac{\partial}{\partial x}\right) L_{x}+\frac{1}{\rho_{c}} L_{\delta}\right\} \text {. }
$$

On inserting Eqs. (86) and (102) into Eq. (84), one obtains

$$
\begin{aligned}
\Phi(\vec{x})= & \phi^{(0)}(\vec{x})+\frac{1}{\sqrt{2 \pi}} \\
& x \sum_{m^{\prime} n^{\prime}} e^{i m^{\prime} \phi} Q_{m^{\prime} n^{\prime}}(n) x_{m n^{\prime} m^{\prime} n^{\prime}}(x),
\end{aligned}
$$

where the incident wave is the $(m, n)$ mode (see Eq. (82)), and

$$
\begin{aligned}
& x_{m n ; m^{\prime} n^{\prime}}(x)= \\
& x \frac{1}{2 \pi} \int_{0}^{2 \pi} d \phi_{0} \int_{0}^{1} n_{0} d n_{0} \int d x_{0} e^{-i m^{\prime} \phi_{0}} Q_{m^{\prime} n^{\prime}}\left(n_{0}\right) \\
& x g_{m^{\prime} n^{\prime}}\left(x_{,} x_{0}\right) L_{s} e^{i m \phi_{0}} Q_{m n}\left(n_{0}\right) R(0)\left(x_{0}\right) .
\end{aligned}
$$

For an axisymmetric flow, $L_{s}$ is independent of $\phi_{0}$ thus one can readily obtain

$$
\begin{aligned}
x_{m n ; m^{\prime} n^{\prime}}=\delta_{m m^{\prime}} & x_{n n^{\prime}}^{m} \\
x_{n n^{\prime}}^{m}=\int n_{0} d n_{0} \int & d x_{0} Q_{m n^{\prime}}\left(n_{0}\right) g_{m n^{\prime}}\left(x, x_{0}\right) \\
& \times L_{s} Q_{m n^{\prime}}\left(n_{0}\right) R^{(0)}\left(x_{0}\right) .
\end{aligned}
$$

Furthermore, if $\rho_{\delta}, P_{\delta}$ and $U_{\delta}$ are independent of $n$ and if $\hat{n} \cdot \vec{U}_{\delta}=0$, one has then

$$
\begin{aligned}
& x_{n n^{\prime}}^{m}=\delta_{n n^{\prime}} x_{n n}^{m}(x), \\
& x_{n n}^{m}=\int d x_{0} g_{m n}\left(x, x_{0}\right) L_{s} R^{(0)}\left(x_{0}\right) .
\end{aligned}
$$

It follows that mode conversion, from one mode to another, occurs only if the transversal gradients of the steady fluid variables are nonnegligible. 3

\section{Power Reflection and Transmission Coefficients}

The acoustic power intensity in the isentropic flow is given by 4

$$
\begin{aligned}
I=\langle p \vec{u}\rangle+\vec{U}\left(\left\langlep^{2}>/ \rho_{0} c^{2}+\right.\right. & \cdot\langle p \vec{u}\rangle / c) \\
& +\rho_{0}\langle(\vec{U} \cdot \vec{u}) \vec{u}\rangle,
\end{aligned}
$$


where < > stands for the time average. The total power across the duct is obtained as

$$
K=\int \tilde{I} \cdot \hat{n} d A
$$

where $d A$ is the surface area element and $\hat{n}$ the unit vector normal to the surface. In the uniform duct element containing the axisymmetric mean flow, we have

$$
K=2 \pi \int I_{x} h d h
$$

with the axial component $I_{x}$ of the power intensity given by

$I_{x}=\left\langle p u_{x}\right\rangle\left(1+m^{2}\right)+p_{0} u\left(\left\langle u_{x}^{2}\right\rangle+\left\langle p^{2}\right\rangle o_{0}^{2} c^{2}\right)$.

Let $K_{i}, K_{r}$ and $K_{t}$ denote the total

powers respectively of the incident, the reflected and the transmitted wave. The power reflection and reflection coefficients, $R$ and $T$, are defined as

$$
\begin{aligned}
& R=K_{r} / K_{j}, \\
& T=K_{t} / K_{j} .
\end{aligned}
$$

Using Eqs. (71), (81), (76) and (77), one can obtain

$$
\begin{aligned}
& R=\frac{\cosh \left[\pi\left(k_{+}-k_{-}\right) a / \varepsilon\right]+\cosh (\pi \sigma)}{\cosh \left[\pi\left(k_{+}-k_{-}\right) a / \varepsilon\right]+\cosh (\pi \sigma)} \\
& T=\frac{2 \sinh \left(\pi k_{+} a / \varepsilon\right) \cdot \sinh \left(\pi k_{-} a / \varepsilon\right)}{\cosh \left[\pi\left(k_{+}+k_{-}\right) a / \varepsilon\right]+\cosh (\pi \sigma)}
\end{aligned}
$$

Note that $R+T=1$. That is, the acoustic energy is conserved as it shoula be in a linear analysis of the acoustic field. The terms neglected in the approximation would not create or annihilate the acoustic energy. They, if included, might transfer the energy between modes or between the reflected and the transmitted waves. Also note that Eqs. (116) and (117) are similar to Eqs. (25) and (26) in Ref. 1. The mean flow dependence is included only through the parameters $k_{ \pm}, \varepsilon$ and $\sigma$. The latter are even functions of $M$. Thus the power reflection and transmission coefficients are incependent of the mean flow direction, the positive or the negative x-direction.

Numerical results will be discussed in terms of the acoustic power transmission loss (TL) in the circular cosh ducts. The mean flow is isentropic flow and has a uniform steady fluid density. As mentioned earlier, a uniform steacy density is a good approximation for low Mach number flow in inlet ducts. Numerical calculation includes the Mach numbers up to the value of 0.5 at the duct throat.

The TL is defined as

$$
\text { - TL }=-10 \log _{10}(T)
$$

For the present calculation, Eq. (117) is used for $T$. The TL is plotted as a function of the throat Mach number $M_{0}$ or of the frequency parameter $\gamma_{-}$or $\zeta$. The parameter $\gamma_{-}$is the mode cut-off ratio referenced to the left hand side uniform duct element, and is referred to as the inlet cut-off ratio:

$$
r_{-}=k b_{-} / \alpha \sqrt{1-M_{-}^{2}} \text {. }
$$

The inlet cut-off ratio is a convenient parameter characterizing the modes contained in the incident wave. The sound generated in a fan duct often comprises many different modes. In such case, the mode distribution can be obtained as a function of the cut-off ratio. Another cut-off ratio useful for discussion is that referenced to the duct throat, given by

$$
\gamma_{0}=k b_{0} / \alpha \sqrt{1-M_{0}^{2}} .
$$

If $\gamma_{0}>1$, the mode is cut-on through the entire duct. On the other hand, if $r_{0}<1$, the mode that is cut-on initially $\left(\gamma_{-}>1\right)$, is cutoff in the converging-diverging section. Except for low frequencies $\left(k b_{0}<3\right)$, the TL is approximately $3 \mathrm{~dB}$ for $\gamma_{0}=1$, independent of the duct geometry or the Mach number. The two cut-off ratios are related to each other as

$$
\gamma_{-}=\gamma_{0} \beta_{-}\left[\left(1-M_{0}^{2}\right)\left(1-M_{0}^{2} B_{-}^{4}\right)\right]^{1 / 2}
$$

The parameter $\zeta$ is defined in Eq. (53), and can be written as

$$
\zeta=\frac{k b_{0}}{1-m_{0}^{2}}\left(1-\frac{1}{\gamma_{0}}\right) \text {. }
$$

This parameter collapses the TL curves for many different modes.

In Fig. 3, the TL aivided by $k b_{-}$is plotted as a function of $\gamma_{-}$for the various values of $M_{0}$ between 0 and 0.5 , and for $B_{-}=1.1$ or 1.3 . The other duct parameters are $a / b_{-}=1, \zeta=1$. On the top of the figure are shown the arrow marks, each pointing to a value of $\gamma_{\text {- }}$ which corresponds to $\gamma_{0}=1$ with the given value of $\mathrm{M}_{0}$ (cf. Eq. (121). The $(8,5)$ mode has been used for the calculation. However, except in the vicinity of $\gamma_{0}=1$, the results may be used for modes with eigenvalue from 8 to 25 , within an error of $1 \mathrm{~dB}$. As expected, the $T L$ decreases with the increasing value of $\gamma_{-}$ (see Ref. 1). The cecreasing rate of the $\mathrm{TL}^{-}$is faster for larger Mach number. For a fixed value of $\gamma_{-}$, the TL is smaller for larger Mach number. Since, TL/kb, is plotted in Fig. 4, TL itself is proportional to $k b_{-}$for a fixed value of $\gamma_{-}$. It follows that, for a mode distribution given as a function of the inlet cut-off ratio, the converging-diverging auct contour reduces the transmitted sound more effectively for cases when the distribution comprises modes of the larger eigenvalues. 
In Fig. 4, the $T L / k b_{-}$is plotted as a function of $M_{0}$, for fixed values of $\gamma_{-}$. As $M_{0}$ increases, the TL decreases very slowly in the beginning $\left(M_{0} \cong 0\right)$, and then decreases at a gradually increasing rate until $M_{0}$ reaches a value for which $r_{0}=1$. This mean flow dependence of the TL is directly related to the change of the cut-off ratio at the throat. As one can notice from Eq. (112), ro increases with increasing $M_{0}$ for $\gamma_{-}$held constant.

In Fig. 5, the $T L$ is plotted as a function of $\zeta$. Each curve, for a value of $\mathrm{M}_{0}$, includes many modes with eigenvalues greater than 5. The $T L$ increases with increasing $\zeta$, and becomes approximately $3 \mathrm{~dB}$ at $\zeta=0$ independent of the Mach number and the duct parameters. The advantage of this presentation is that almost all the modes are subject to the same TL for a given value of $\zeta$. The mean flow dependence of the TL in this figure appears misleading. However, note that, with $M_{0}$ increased, ro increases and $\mathrm{kb}_{0}$ should be decreased to keep $\zeta$ unchanged (see Eq. (122)).

\section{Concluding Remarks}

In an attempt to improve the understanding of the acoustic characteristics of a fan duct system, mode propagation has been investigated in a particular class of converging-diverging circular ducts - circular cosh ducts, with isentropic and inviscid mean flow. The auct shape can be adjusted by means of three duct parameters, and covers a wide range of coverging-diverging ducts of practical interest.

On the assumption that the duct cross section area varies slowly, an approximate wave equation has been derived. The equation is divided into two parts: (1) the homogeneous wave equation involving the mean flow of uniform steady density fluid, and (2) the interaction between the acoustic field and the deviation of the steady fluid variables from the constant steady density fluid flow. The homogeneous equation is solved in a closed form, and the interaction term is treated by means of an iteration-perturbation method. The solution of the homogeneous equation is regarded a good approximation in cases of low Mach number mean flow.

With the interaction neglected, a mode is preserved, and the acoustic power transmission coefficient increases with the mean flow increase. This mean flow effect is directly related to the change of the cut-off ratios: With the increasing mean flow, the cut-off ratio at the duct throat increases faster than at the uniform duct sections (inlet and exit). For given duct geometry and mean flow, the TL of many different modes can be made to collapse onto a single curve with a proper choice of frequency parameter $(\zeta)$. The mean flow effects remain unchanged with the flow reversed. The interaction term needs numerical computations for its detailed discussions. However, the formal solution shows that a mode may be converted to others only if the transverse gradients of the steady fluid variables are not negligible.

\section{References}

1. Cho, Y.-C. and Ingard, K. U., "Higher Order Mode Propagation in Nonuniform Circular Ducts," AIAA Paper 80-1018, June 1980.

2. Morse, P. M. ana Feshbach, H., Methods of Theoretical Physics, McGraw-HilT Book Co., New York, 1953.

3. Tam, C. K. W., "Transmission of Spinning Acoustic Modes in a Slightly Nonuniform Duct," Journal of Sound and Vibration, Vol. 18, No. 3, 0ct. 1971, pp. 339-351.

4. Morfey, C. L., "Acoustic Energy in Nonuniform Flow," Journal of Sound and Vibration, Vol. 14, No. 2, Jan. 1971, pp. 159-170.

TABLE 1. - VALUE OF $\varepsilon$

\begin{tabular}{|c|c|c|c|c|c|c|}
\hline \multirow[t]{2}{*}{$B_{-}$} & \multirow[t]{2}{*}{$M_{0}$} & \multirow[t]{2}{*}{ Eigenvalue } & \multicolumn{4}{|c|}{$\zeta$} \\
\hline & & & -0.4 & -0.2 & 0 & 0.2 \\
\hline \multirow{2}{*}{1.1} & 0.2 & $\begin{array}{r}4.20 \\
8.54 \\
16.53 \\
23.27\end{array}$ & $\begin{array}{l}1.0020 \\
1.0017 \\
1.0016\end{array}$ & $\begin{array}{l}1.0020 \\
1.0017 \\
1.0015 \\
1.0015\end{array}$ & 1 & $\begin{array}{l}1.0007 \\
1.0010 \\
1.0012 \\
1.0012\end{array}$ \\
\hline & 0.4 & $\begin{array}{r}4.20 \\
8.54 \\
16.53 \\
23.27\end{array}$ & $\begin{array}{r}0.9980 \\
.9953 \\
.9944\end{array}$ & $\begin{array}{r}0.9952 \\
.9937 \\
.9932\end{array}$ & $\downarrow^{0.9920}$ & $\begin{array}{r}0.9845 \\
.9886 \\
.9903 \\
.9908\end{array}$ \\
\hline \multirow{2}{*}{1.3} & 0.2 & $\begin{array}{r}4.20 \\
8.54 \\
16.53 \\
23.27\end{array}$ & $\begin{array}{l}1.0039 \\
1.0025 \\
1.0019 \\
1.0017\end{array}$ & $\begin{array}{l}1.0026 \\
1.0019 \\
1.0015 \\
1.0014\end{array}$ & 1.0012 & $\begin{array}{l}0.9997 \\
1.0004 \\
1.0008 \\
1.0009\end{array}$ \\
\hline & 0.4 & $\begin{array}{r}4.20 \\
8.54 \\
16.53 \\
23.27\end{array}$ & $\begin{array}{r}1.0041 \\
.9946 \\
.9897 \\
.9880\end{array}$ & $\begin{array}{r}0.9948 \\
.9895 \\
.9868 \\
.9860\end{array}$ & 0.9839 & $\begin{array}{r}0.9710 \\
.9778 \\
.9808 \\
.9817\end{array}$ \\
\hline
\end{tabular}

TABLE 2. - VALUE OF $\&$ AT $\zeta=0$

\begin{tabular}{|r|r|r|r|r|r|}
\hline \multirow{2}{*}{ Mo } & \multicolumn{5}{|c|}{$B_{-}$} \\
\cline { 2 - 6 } & 1.1 & 1.2 & 1.3 & 1.4 & 1.5 \\
\hline & & & & & \\
0.1 & 1.0004 & 1.0006 & 1.0005 & 1.0004 & 1.0002 \\
.2 & 1.0014 & 1.0015 & 1.0012 & 1.0005 & .9998 \\
.3 & 1.0008 & 1.0001 & .9987 & .9971 & .9954 \\
.4 & .9920 & .9875 & .9837 & .9806 & .9776 \\
.5 & .9438 & .9316 & .9271 & .9244 & .9223 \\
\hline
\end{tabular}




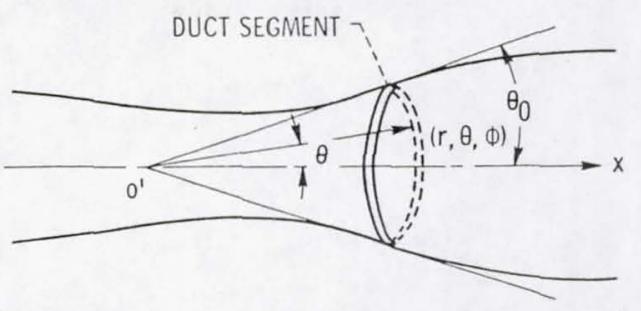

Figure 1. - Duct segment and local spherical coordinate system.

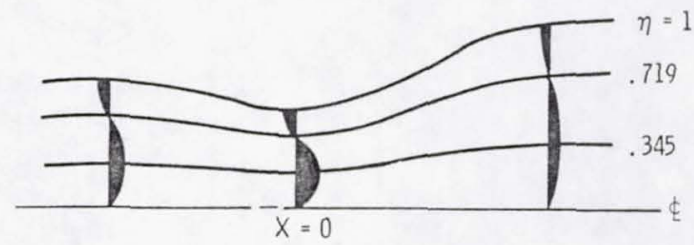

Figure 2. - Coordinate surfaces $(\eta=0.345,0.719,1)$ and transversal shape of $(1,1)$ mode at various axial locations.

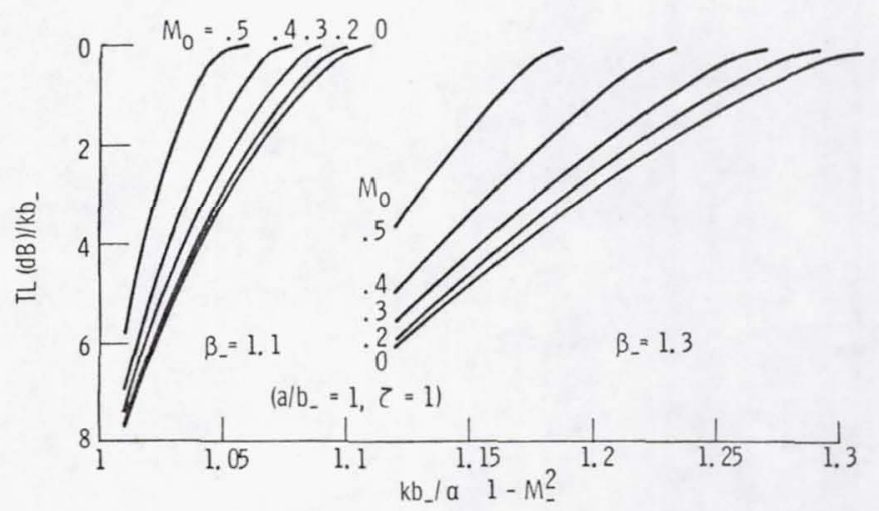

Figure 3. - Power transmission loss (TL) of (8.5) mode, divided by $\mathrm{kb}_{-}$, versus inlet cutoff ratio $\left(\gamma_{-}\right)$. 


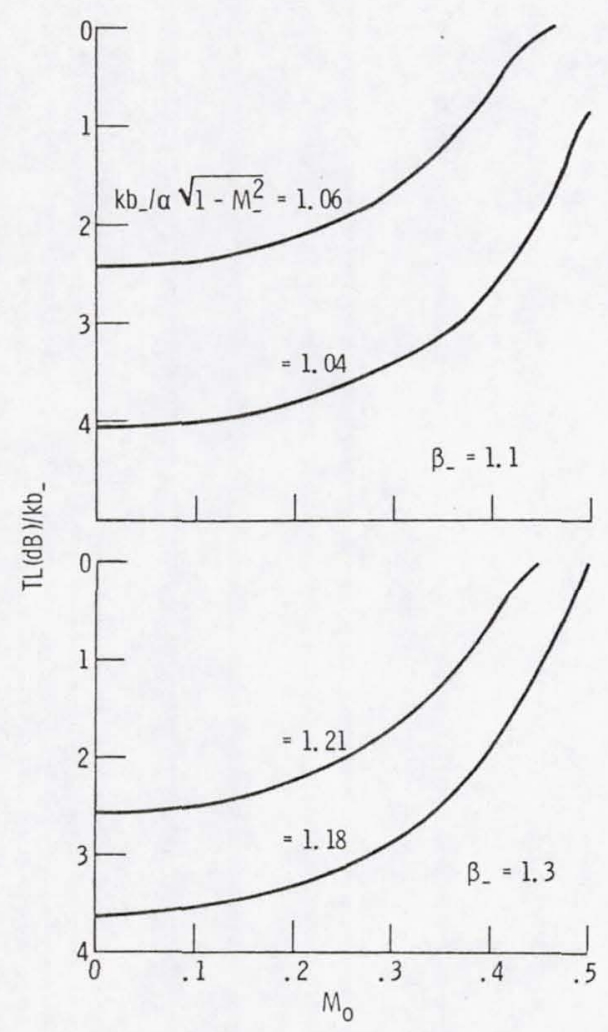

Figure 4. - Power transmission loss (TL) of (8.5) mode, divided by $k b_{-}$, versus Mach number at throat $\left(M_{0}\right)$.
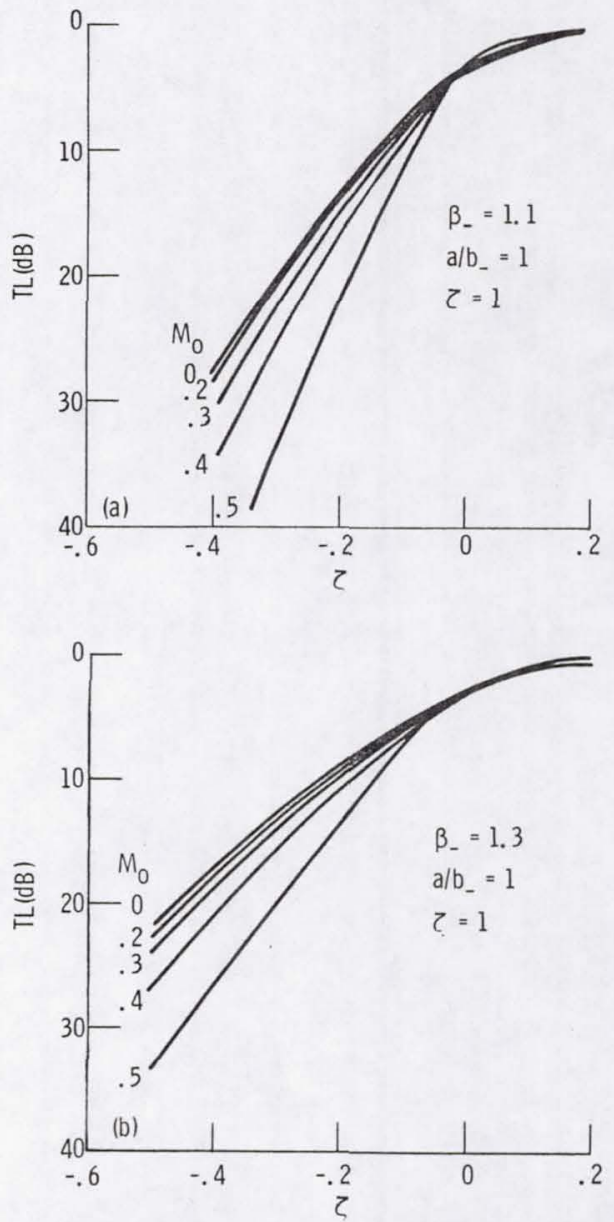

Figure 5. - Power transmission loss (TL) versus $\zeta$. Each curve representing modes with eigenvalue greater than 3 . 


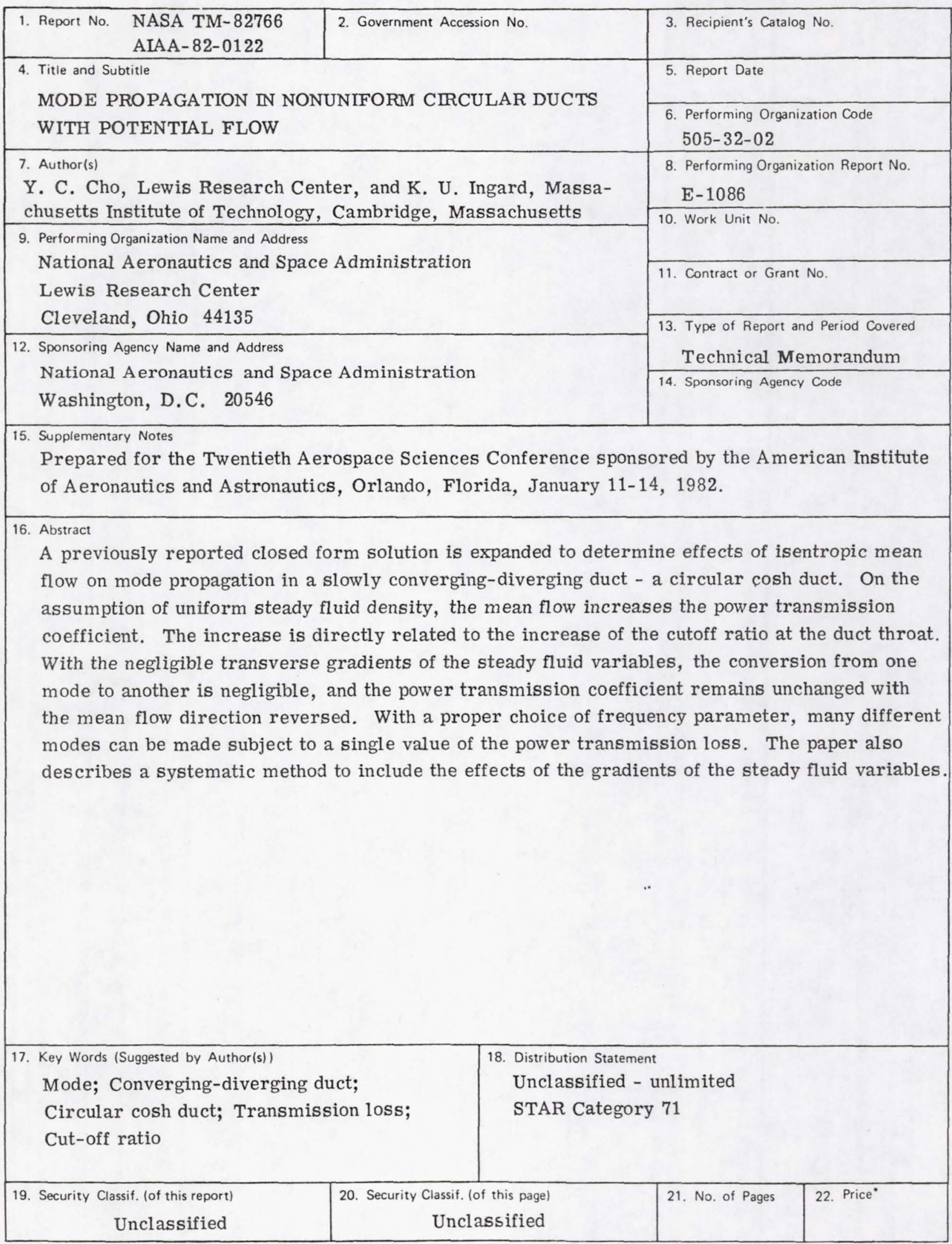

* For sale by the National Technical Information Service, Springfield, Virginia 22161 